\title{
Aménagement du Var inférieur et protection des nappes souterraines : un exemple d'extraction contrôlée de graviers
}

PAR

\section{P. Prudhomme}

Président Directeur général de Géohydraulique

Directeur général du LCHF

Notre exposé a pour but de présenter l'aménagement réalisé sur le lit inférieur du Var. Cet aménagement devait répondre à un double objectif :

- relever les niveaux phréatiques de la nappe alluviale environnante qui s'étaient abaissés progressivement au cours des dernières années;

- permettre la poursuite de l'extraction de graviers, particulièrement intéressante pour les constructions de la région de Nice.

Cet aménagement a fait l'objet d'une présentation générale, publiée dans la revue Travaux en août-septembre 1972 , sous la signature de $\mathrm{M}$. Pradon, Ingénieur des Ponts et Chaussées chargé de l'Arrondissement des Ports Maritimes et Aériens et du Service Hydraulique à la Direction Départementale de l'Equipement et du Logement des Alpes-
Maritimes. Cet article présentait l'ensemble des aspects relatifs aux travaux d'aménagement réalisés ou en cours d'exécution, alors que notre exposé se limitera au seul domaine de l'hydraulique souterraine, pour lequel une étude préalable à ces aménagements a été conduite par Géohydraulique en liaison étroite avec les services du Bureau de Recherches Géologiques et Minières.

Dans le cadre de cet exposé, après avoir analysé les données du problème tel qu'il se présentait à l'origine, nous préciserons les études réalisées en matière de prévision des effets des aménagements sur la nappe alluviale, puis nous présenterons le schéma du dispositif retenu pour cet aménagement et nous terminerons par un examen des informations recueillies à la suite de la réalisation des premiers travaux commencés dès 1971 .

\section{1 - Présentation du problème}

C'est en 1967, à la suite d'une période de sécheresse estivale, que la situation de la nappe alluviale devint particulièrement préoccupante: certains puits, utilisés pour l'irrigation de terrains agricoles riverains du Var, se trouvèrent taris.

En fait, l'abaissement progressif de la nappe alluviale se manifestait depuis plusieurs années et les pouvoirs publics portaient attention à ce problème, ce qui leur a permis de réagir rapidement dès l'apparition de ces tarissements de puits.

Depuis de nombreuses années, les derniers kilomètres du fleuve (du vallon de Lingostrière à l'embouchure, soit environ $6 \mathrm{~km}$ ) étaient soumis à d'importantes extractions de graviers qui constituaient les principaux matériaux concassables du département, utilisés même fréquemment du fait du faible coût d'extraction, sans traitement préalable, pour la constitution de remblais. Ces extractions combinées, semble-t-il, à une diminution sensible, au cours des dernières décennies, des apports solides du fleuve par saltation, ainsi qu'à une tendance naturelle du lit du Var à l'approfondissement, s'étaient traduites par un abaissement du fond du lit atteignant par endroits 4 à $5 \mathrm{~m}$. Corrélativement, il entraînait un abaissement de la ligne d'eau du fleuve et, par conséquent, celui de la surface libre de la nappe alluviale environnante pour laquelle le Var constitue le niveau de base.

Afin de limiter les effets de l'extraction, les exploitants de graviers, sous l'impulsion du Professeur Mangin, ont procédé eux-mêmes à l'aménagement de seuils maintenant artificiellement le plan d'eau à une cote supérieure à celle résultant d'un écoulement libre. Mais, outre le fait que 
ces seuils étaient peu élevés, leur construction sommaire les rendait très vulnérables aux crues subites du Var qui les détruisaient régulièrement, et l'on observait immédiatement un abaissement de la surface piézométrique à proximité dudit seuil.

L'attention a d'abord été portée sur la partie inférieure du Var de $6 \mathrm{~km}$ en aval du vallon de Lingostrière où les extractions de graviers avaient eu les effets néfastes évoqués.

Dans cette zone, les caractéristiques principales étaient les suivantes:

- largeur du lit du Var : $300 \mathrm{~m}$ environ;

- extension latérale de la nappe alluviale:

- $800 \mathrm{~m}$ à $900 \mathrm{~m}$ en rive gauche,

- extension très limitée en rive droite;

- pente du lit du Var: entre 4,6 et 6,2 pour 1000 ;

- épaisseur d'alluvions: de l'ordre d'une trentaine de mètres pour la partie en relation directe avec le fleuve;

- pente moyenne du mur des alluvions : estimés à 5/1000 soit équivalente à la pente moyenne du lit du fleuve;

- perméabilité des alluvions: les résultats d'essais de pompage, interprétés par la Compagnie Générale des Eaux, indiquaient les valeurs suivantes:

$$
\begin{gathered}
K==5 \times 10^{-3} \mathrm{~m} / \mathrm{s} \text { à } K=3 \times 10^{-2} \mathrm{~m} / \mathrm{s} \\
T=5 \times 10^{-2} \mathrm{~m}^{2} / \mathrm{s} \text { à } T=12 \times 10^{-2} \mathrm{~m}^{2} / \mathrm{s} \\
\text { (valeurs affectées par des hétérogénéités verticales } \\
\text { non continues) }
\end{gathered}
$$

- débit d'étiage du Var: $10 \mathrm{~m}^{3} / \mathrm{s}$ enregistré en 1967 (étiage considéré comme très sévère);

- pompages dans la nappe en bordure du Var:

- puits CGE en rive gauche, tout à fait dans la partie aval;

- puits SIEVI et SILRDV en rive droite, à mi-distance entre Lingostrière et l'embouchure du fleuve.

Dans le but de satisfaire aux besoins de prélèvements dans la nappe pour les irrigations, il fut d'abord envisagé de procéder à une campagne d'approfondissement des puits. Toutefois, outre le fait que la procédure de mise en œuvre d'une telle campagne était délicate, cette disposition n'apportait aucune solution à deux risques importants :

- dégradation des berges du Var, du fait de l'approfondissement du lit;

- intrusion d'cau salée vers les puits CGE, du fait de l'abaissement de la ligne d'eau du Var et de la nappe alluviale à l'aval.

C'est ainsi que le Service de I'Hydraulique de la Direction Départementale de l'Equipement des Alpes-Maritimes, travaillant en étroite liaison avec la Direction Départementale de l'Agriculture et les différentes administrations et collectivités concernées par le problème, a reçu mission d'étudier un aménagement du lit du Var au moyen de seuils stables implantés dans le cours du fleuve. On reprenait par-là l'idée lancée par le Professeur Mangin, dont le but est de rendre le fil d'eau du fleuve indépendant de la cote du lit (fig. 1). Afin d'assurer le moindre cout des ouvrages, le noyau des seuils devait être constitué par les matériaux mêmes du lit du Var.

Les études ont alors été lancées dans deux directions principales :

- caractéristiques à donner aux seuils pour assurer leur stabilité aux crues (pointes de crue estimées : annuelle
$910 \mathrm{~m}^{3} / \mathrm{s}$, centenaire : $2000 \mathrm{~m}^{3} / \mathrm{s}$ ), et conséquences sur le lit du fleuve en aval des seuils; ces études ont été conduites par le Laboratoire National d'Hydraulique de Chatou;

- évaluation des risques de fuites au droit des seuils en période d'étiage et prévision des effets de l'aménagement des seuils sous la nappe alluviale; ces études d'hydraulique souterraine ont été conduites par GEOHYDRAULIQUE en étroite liaison avec le BRGM.

Ce sont ces dernières études qui font seules l'objet du développement ci-dessous.

\section{2 - Etudes d'hydraulique souterraine réalisées}

Les études conduites au Laboratoire de Chatou montraient qu'il était possible de réaliser, de façon économique, des seuils stables arasés à la cote $+4 \mathrm{~m}$ au-dessus du lit moyen du Var, tel qu'il apparaissait fin 1967. C'est sur cette hypothèse qu'ont été lancées les études d'hydraulique souterraine dans les deux directions suivantes:

- évaluation du débit de fuite au droit du seuil : pour assurer un relèvement réel du fil de l'eau à l'amont du seuil, en période d'étiage, ce débit de fuite ne devait pas être supérieur à $10 \mathrm{~m}^{3} / \mathrm{s}$ (étiage enregistré en 1967);

- prévision de l'effet des seuils sur le relèvement de la surface libre de la nappe alluviale.

\section{1 - ÉTUdE DU DÉBIT DE FUITE}

Cette étude a d'abord porté sur l'examen du dèbit de fuite direct au travers et sous le seuil, en s'intéressant particulièrement à l'effet de diverses caractéristiques du seuil lui-même. Ensuite, en même temps que la prévision des effets des seuils sur la nappe alluviale, le débit de fuite total (direct + - latéral) (fig. 2) a été évalué.

\subsection{1 - Incidence de la conception des} seuils sur le débit de fuite direct

A l'aide d'un modèle analogique électrique (papier conducteur), on a représenté une tranche verticale d'aquifère (fig. 3) surmontée du seuil et perpendiculaire à celui-ci.

Dans cette étude, différents paramètres ont été pris constants et les valeurs suivantes, résultant des données existantes ou des essais réalisés, leur ont été attribuées :

- perméabilité moyenne $K=2 \times 10^{-2} \mathrm{~m} / \mathrm{s}$ (cette valeur était associée à une épaisseur équivalente moyenne maximale de $20 \mathrm{~m}$ );

- forme du seuil : talus amont de pente $1 / 1$, talus aval de pente $1 / 2$;

- hauteur de la retenue en amont du seuil : $4 \mathrm{~m}$ au-dessus du niveau moyen du lit du Var;

- longueur du seuil : $160 \mathrm{~m}$ (celle-ci résultait du profil du lit au droit du projet de seuil envisagé).

Par contre, d'autres paramètres ont donné lieu à l'étude 


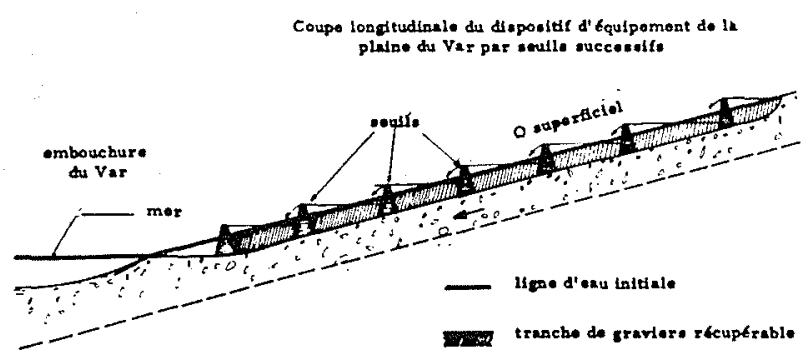

1/ Principe de l'aménagement par seuils.

2/ Schéma en perspective de l'écoulement dans l'aquifère à proximité d'un seuil.

\section{3/ Simulation du débit de fuite direct à travers}

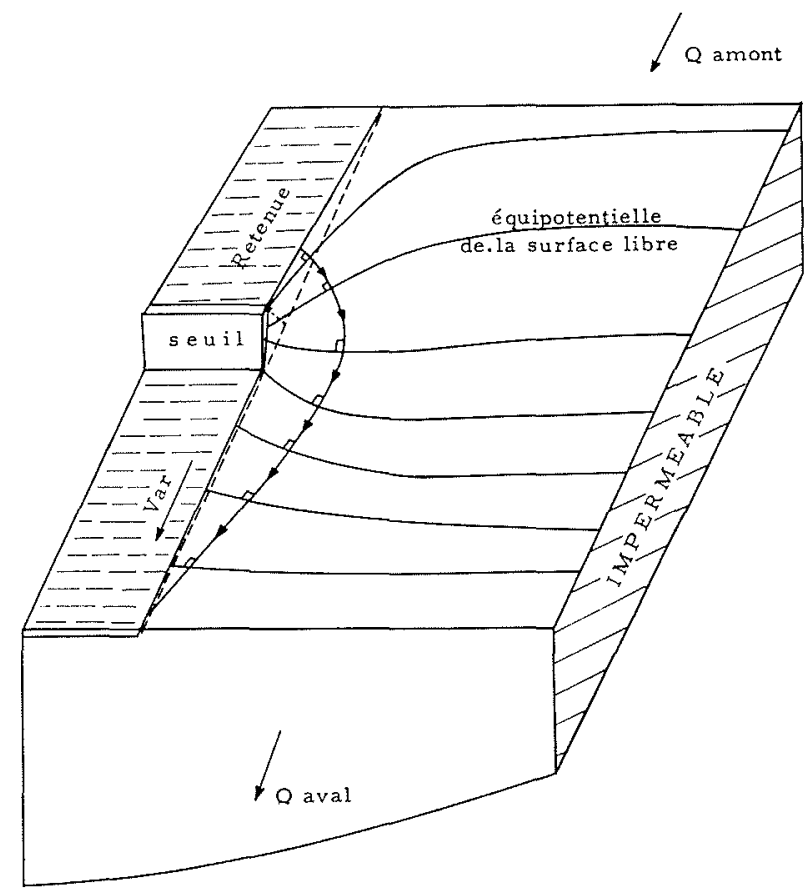
et sous un seuil.

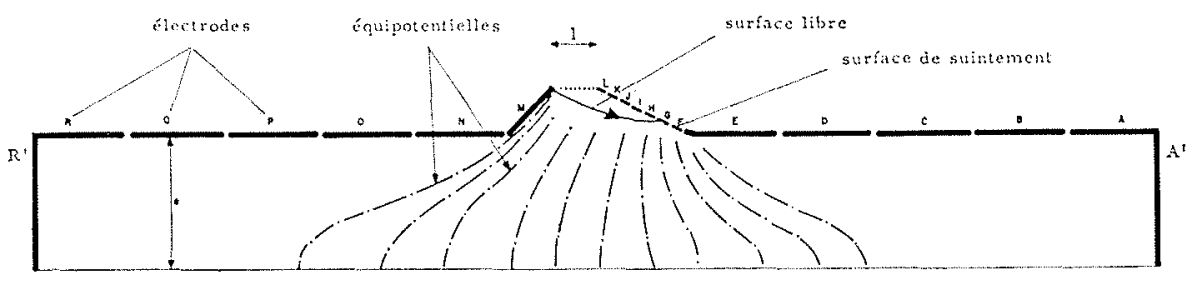

de plusieurs valeurs afin de déterminer leur influence sur le débit de fuite:

- épaisseur de l'aquifère $e$ sous le seuil : $e=12 \mathrm{~m}$ et $e=20 \mathrm{~m}$;

- largeur $l$ du seuil en tête : $l=4 \mathrm{~m}$ et $l=8 \mathrm{~m}$;

- nature du seuil:

- seuil perméable $K=2 \times 10^{-2} \mathrm{~m} / \mathrm{s}$,

- seuil étanche,

- parement amont du seuil étanche et rideau de palplanches pénétrant de $5 \mathrm{~m}$ dans l'aquifère.

Les résultats, concernant le débit de fuite direct et portant sur ces divers cas, sont résumés dans le tableau ci-dessous :

\begin{tabular}{|c|c|c|c|c|}
\hline CAS & $\begin{array}{l}\text { EPAISSEUR } \\
\text { D'AQUIFÈRE }\end{array}$ & $\begin{array}{l}\text { LARGEUR } \\
\text { DU SEUIL } \\
\text { EN TÊTEE }\end{array}$ & Nature du SEUIL & $\begin{array}{l}\text { DÉBIT } \\
\text { en } \\
\mathrm{m}^{3} / \mathrm{s}\end{array}$ \\
\hline $\mathrm{n}^{\circ} 1$ & $12 \mathrm{~m}$ & $4 \mathrm{~m}$ & perméable & 8 \\
\hline$n^{\circ} 2$ & $20 \mathrm{~m}$ & $4 \mathrm{~m}$ & perméable & 9,8 \\
\hline $\mathrm{n}^{\circ} 3$ & $20 \mathrm{~m}$ & $8 \mathrm{~m}$ & perméable & 8,6 \\
\hline$n^{\circ} 4$ & $20 \mathrm{~m}$ & $8 \mathrm{~m}$ & en matériaux étanches & 6,4 \\
\hline$n^{\circ} 5$ & $20 \mathrm{~m}$ & $4 \mathrm{~m}$ & $\begin{array}{l}\text { parement amont étanche } \\
+ \text { rideau palplanches } \\
\text { amont }\end{array}$ & 6 \\
\hline
\end{tabular}

De ces résultats, on a pu tirer les conclusions suivantes: - l'accroissement de largeur du seuil ne présente que peu d'intérêt;

- la construction du seuil en matériaux étanches apporte une réduction limitée à $25 \%$ du débit de fuite : on peut penser que le colmatage provoqué par la sédimentation des éléments fins en amont de l'ouvrage aura sensiblement les mêmes effets;

- le parement amont étanche, doublé d'un rideau de palplanches, serait la solution la plus efficace (réduction de $39 \%$ du débit de fuite) s'il s'avérait nécessaire de limiter ce débit de fuite direct.

Quoi qu'il en soit, il apparaissait que sur un plan global le débit de fuite direct n'était pas considérablement inférieur au débit d'étiage possible du Var de $10 \mathrm{~m}^{3} / \mathrm{s}$. En conséquence, il fut décidé de procéder à des études plus complexes permettant de tenir compte d'une perméabilité moins grande des terrains en place, par rapport à la perméabilité escomptée du seuil, et intégrant l'ensemble du débit de fuite (direct et latéral par l'aquifère adjacent).

\subsection{2 - Evaluation du débit de fuite global}

Cette étude a été conduite parallèlement à la prévision de l'influence des seuils sur le niveau piézométrique de la nappe alluviale. Elle a été menée en mettant en œuvre des moyens relativement complexes, car il était indispensable de prendre en considération le caractère tridimensionnel de 


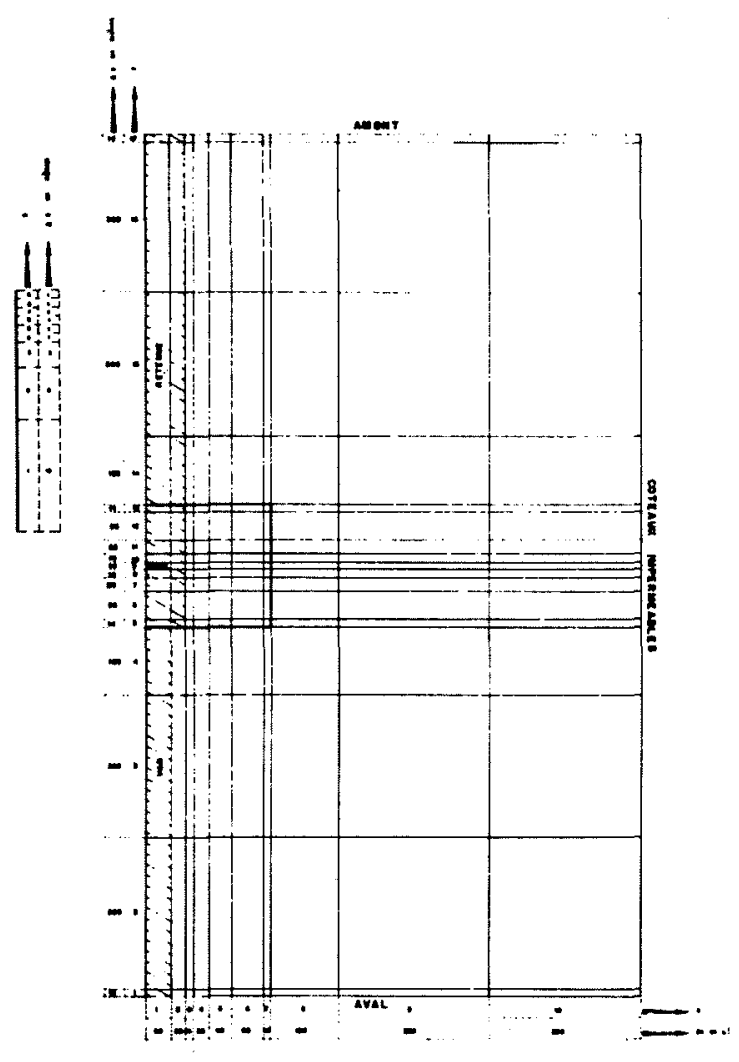

4/ Limites d'extension et découpage des modèles mathématiques.

l'écoulement dont l'influence pouvait être particulièrement importante, à proximité des seuils, tant sur le débit de fuite latéral que sur les niveaux piézométriques de la nappe dans cette zone.

Compte tenu de cette remarque, le choix s'est porté sur l'utilisation de modèles mathématiques tridimensionnels aptes à simuler l'écoulement dans une nappe à surface libre.

De tels modèles mathématiques nécessitent un découpage spatial qui soit le plus en harmonie possible avec les lignes de courant qui résulteront des différentes hypothèses testées. D'autre part, la finesse du découpage influe directement sur le nombre de mailles du modèle et par répercussion sur le temps de calcul sur ordinateur (donc sur le coût des études). De ce fait, un modèle bidimensionnel simple (papier conducteur électrique) a été utilisé à titre de dégrossissage pour permettre de prévoir la forme des lignes de courant dans la nappe résultant de l'aménagement du seuil. C'est sur les indications fournies par ce modèle que le découpage des modèles mathématiques tridimensionnels a été défini.

\subsubsection{1 - ZONE REPRÉSENTÉE PAR LES MODÈLES}

L'hypothèse de seuils arasés à la cote $+4 \mathrm{~m}$ au-dessus du niveau moyen du lit du Var ayant été retenue, l'objectif était de représenter tout un domaine moyen de la nappe alluviale pouvant être influencé par un tel seuil. En considérant une pente de $5 / 1000$ du lit du fleuve, la retenue engendrée par un seuil de $4 \mathrm{~m}$ de haut devait s'étendre sur $800 \mathrm{~m}$ en amont du seuil. 'Toutes choses étant supposées rester identiques en amont de cette limite de retenue, la limite d'influence possible sur la nappe en amont du seuil se situait donc à cette distance. Par ailleurs, compte tenu de l'extension latérale faible $(800 \mathrm{~m})$ de la nappe alluviale concernée, il est apparu raisonnable de limiter la représentation aval jusqu'à une distance équivalente à celle imposée en amont.

Le domaine représenté par les modèles est schématisé sur la figure 4, son extension en est la suivante :

- limite amont: $800 \mathrm{~m}$ en amont du seuil;

- limite aval : $800 \mathrm{~m}$ en aval du seuil;

- extension latérale nappe: jusqu'aux coteaux supposés imperméables, soit à une distance de $800 \mathrm{~m}$ des berges du Var;

- extension latérale Var : à $120 \mathrm{~m}$ à l'intérieur du cours d'eau par rapport à la digue. Cette limite correspondait à une ligne d'écoulement stable parallèle au lit du fleuve.

\subsubsection{2 - CARACTÉRISTIQUES DES MODÈLES MATHÉMATIQUES UTILISÉS}

Un premier modèle d'ensemble, comprenant 3024 cellules spatiales, s'est avéré trop imprécis malgré les cellules d'espace de faibles dimensions retenues dans la zone du seuil. Cette imprécision venait du trop important rapport existant entre le volume des grandes cellules (zones où les lignes de courant ont des courbures faibles) et celui des petites cellules (zones où les lignes de courant ont de fortes courbures).

Il a donc été décidé de construire deux modèles 《gigognes »:

- modèle 1 , représentant tout le domaine concerné, mais avec un découpage grossier dans la zone centrale voisine du seuil (fig. 4);

- modèle 2 , représentant le domaine limité à la partie centrale voisine du seuil (fig. 4).

a) Caractéristiques du modèle 1

Il comportait 1530 cellules. Le découpage des cellules était effectué selon trois axes perpendiculaires $o x, o y, o z$ :

- ox: perpendiculaire au lit du Var, découpé en 10 intervalles;

- oy: parallèle au lit du Var, découpé en 17 intervalles d'aval en amont: le seuil était représenté par un seul intervalle, les autres intervalles étaient symétriques de part et d'autre;

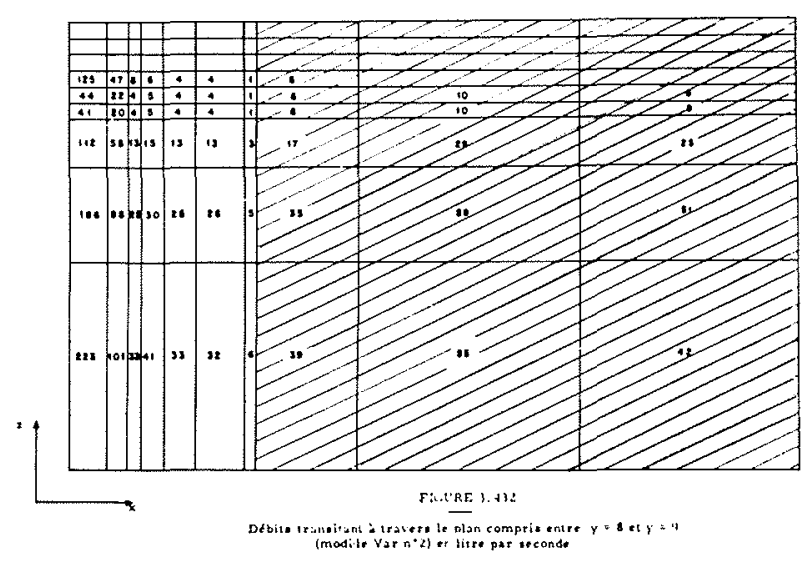

5/ Débit à travers un plan vertical passant par l'axe du seull, calculé par le modèle 1 . 
- oz: vertical, découpé en 9 intervalles, les 6 intervalles supérieurs correspondant à des tranches d'aquifère d'un mètre d'épaisseur seulement (nécessité de la représentation tridimensionnelle).

Le but de ce modèle était double:

- fournir les valeurs recherchées par l'étude (débit de fuite et hauteurs piézométriques) relatives au domaine extérieur à la zone centrale (fig. 4);

- donner les conditions aux limites à imposer au modèle 2 sur le périmètre du domaine central (fig. 4).

\section{b) Caractéristiques du modèle 2}

Il comportait 1881 cellules. Il représentait l'aquifère et le seuil sur un domaine s'étendant :

- sur $228 \mathrm{~m}$ le long du Var, centrés sur le seuil;

- sur $234 \mathrm{~m}$ perpendiculairement au Var, à partir de la ligne d'écoulement stable retenue comme limite à l'intérieur du cours d'eau, soit sur $114 \mathrm{~m}$ en extension latérale de l'aquifère au-delà de la digue du fleuve.

Le découpage des cellules était effectué selon les mêmes trois axes perpendiculaires que pour le modèle 1 :

- ox: découpé en 11 intervalles;

- oy: découpé en 19 intervalles, dont 6 intervalles pour le seuil lui-même;

- oz: découpé en 9 intervalles identiques au modèle 1.

En imposant à ce modèle les conditions aux limites relevées sur le modèle 1 , comme indiqué précédemment, on a obtenu les valeurs du débit de fuite de la retenue et des hauteurs piézométriques prévisionnelles pour le domaine concerné.

\section{c) Valeurs retenues de divers paramètres pour} les deux modèles mathématiques

- Perméabilité : L'usage de modèles mathématiques tridimensionnels a permis de considérer deux valeurs différentes de perméabilité, selon qu'il s'agissait de l'aquifère en place non remanié ou de la zone du seuil (seuil lui-même et ancrage). On aurait pu introduire dans les modèles toute hétérogénéité mais les données existantes ne permettaient pas raisonnablement de formuler des hypothèses valables sur ce point.

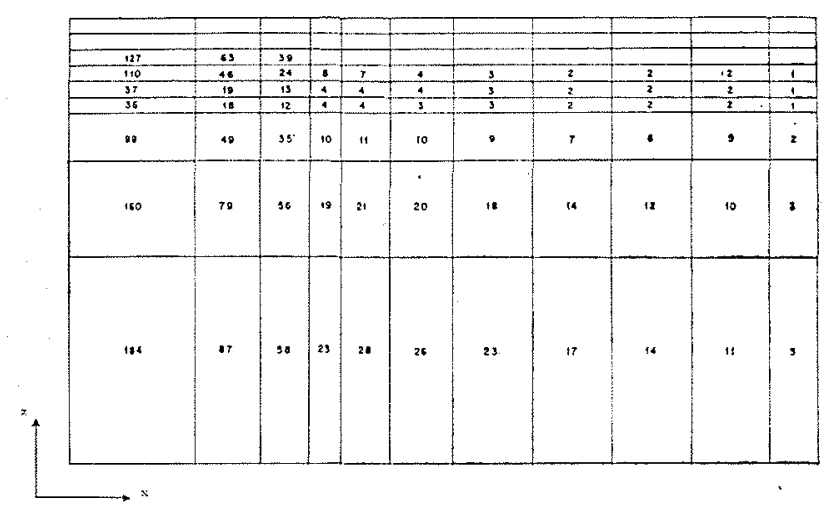

6/ Débit à travers un plan vertical passant par l'axe du seuil, calculé par le modèle 2.
En définitive la perméabilité retenue a été la suivante: - $K=2 \times 10^{-2} \mathrm{~m} / \mathrm{s}$ pour le seuil

- $K=6 \times 10^{-3} \mathrm{~m} / \mathrm{s}$ pour l'aquifère en place non remanié.

- Epaisseur d'aquifère: on a conservé la valeur moyenne de $20 \mathrm{~m}$ sous le niveau moyen du lit du Var.

- Forme du seuil :

- pente amont $1 / 1$

- pente aval $3 / 1$;

- largeur du seuil en tête : $l=5 \mathrm{~m}$;

- hauteur $4 \mathrm{~m}$.

\subsubsection{3 - Débit DE Fuite CAlculé}

Le programme de calcul des modèles mathématiques avait été conçu pour fournir immédiatement les débits traversant la section verticale d'aquifère passant par l'axe du seuil. Ces résultats, cellule par cellule, font l'objet des figures 5 et 6 .

Pour le modèle $\mathrm{n}^{\circ} 1$ (fig. 5) seuls les débits des cellules hachurées sont valables, les valeurs des autres cellules étant obtenues avec plus de précision dans le modèle $n^{\circ} 2$ (fig. 6).

A partir de ces valeurs, on calcule le débit de fuite total possible en tenant compte de la symétrie éventuelle de l'aquifère par rapport à l'axe du Var, ainsi que du débit de fuite direct à travers et sous le seuil dont la valeur unitaire (par mètre linéaire) est fournie par les cellules extrêmes du modèle $\mathrm{n}^{\prime \prime} 2$. Il convient également de retrancher le débit de transit normal de l'aquifère en l'absence de seuil.

Ce calcul a conduit à estimer le débit de fuite total, sur la base des hypothèses retenues, à la valeur de $4,43 \mathrm{~m}^{3} / \mathrm{s}$ sur laquelle le débit de fuite latéral (par la nappe alluviale) intervient pour $17 \%$ du débit total.

De ce résultat, on peut tirer la conclusion que l'aménagement de seuils, même perméables, permettra la création d'un relèvement du fil de l'eau du Var de $4 \mathrm{~m}$ en amont du seuil, même pour un étiage sévère de $10 \mathrm{~m}^{3} / \mathrm{s}$.

\section{2. - ÉTUDE DU RELÈVEMENT DE LA NAPPE ALluViale}

Les hauteurs piézométriques, résultant de l'aménagement d'un seuil, données par les modèles mathématiques, ont permis de déterminer le relèvement de la nappe alluviale sous l'effet du seuil. Pour cela, on a opéré par différence

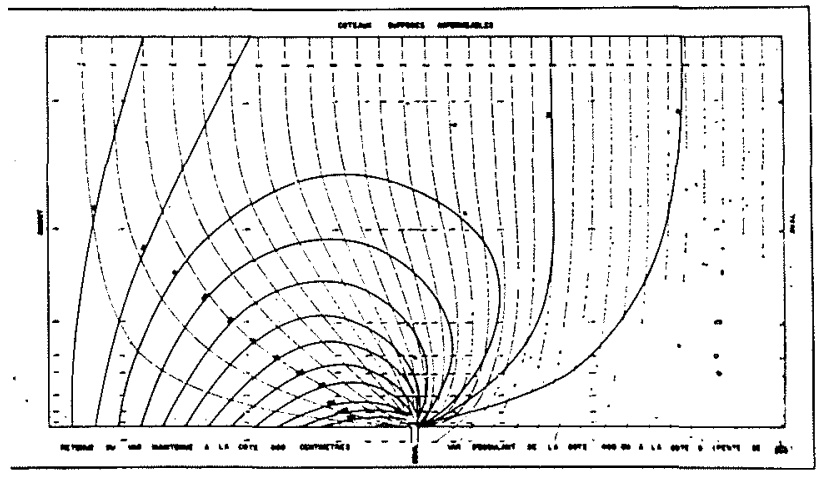

7/ Relèvement prévisionnel de la nappe, calculé à l'alde des modeles mathématiques, sous l'effet de l'aménagement d'un seull. 
entre l'état piézométrique fourni par les modèles et l'état de la nappe sans aménagement supposée soumise à un écoulement linéaire parallèle au Var et de même pente que le cours d'eau.

On constate, figure 7 , que ce relèvement est sensible $(0,75 \mathrm{~m}$ à $450 \mathrm{~m}$ dans la zone du seuil). Comme l'aménagement global doit tendre à l'installation de seuils successifs, les influences des différents ouvrages se superposeront de sorte que le relèvement à en attendre sera nettement supérieur. Les résultats de cette phase d'étude sur un seuil unique, a permis de décider qu'un espacement entre seuils de l'ordre de 800 à $1000 \mathrm{~m}$ devrait conduire à des relèvements intéressants.

Dès le printemps 1968 , il avait été décidé, à titre conservatoire, d’interdire les extractions à l'aval de Lingostrière, où elles étaient pratiquées jusqu'alors, et de ne les autoriser, à l'amont, que sur une épaisseur de $3 \mathrm{~m}$. Il est apparu, toutefois, qu'une telle disposition limiterait à trois années environ la poursuite de l'utilisation des matériaux du Var du fait, notamment, de l'entraînement de ceux-ci, par les crues, vers la zone aval creusée par les extractions antérieures. C'est alors que le Professeur Gévin, intervenant à la demande du Syndicat des Extracteurs du Var, suggéra la réalisation d'extractions par souilles de $1000 \mathrm{~m}$ de long sur $200 \mathrm{~m}$ de large, creusées jusqu'à $6 \mathrm{~m}$ de fond, séparées entre elles par des moles de $500 \mathrm{~m}$ de long où aucune extraction ne serait opérée. L'objectif de cette solution était de conserver, par les moles, des seuils naturels maintenant le fil d'eau à un niveau élevé.

Une étude menée par le Laboratoire de Chatou montra alors que ces moles naturels ne pourraient résister à des crues de l'ordre de $1000 \mathrm{~m}^{3} / \mathrm{s}$ et l'idée semblait devoir être abandonnée. C'est alors que M. Pradon, Ingénieur des Ponts et Chaussées, Chef du Service de l'Hydraulique de la DDE et responsable de l'opération, suggéra de poursuivre l'idée du Professeur Gévin en substituant, aux moles naturels, des seuils du type de ceux envisagés pour la zone en aval de Lingostrière. Ces seuils seraient distants de 800 à $1000 \mathrm{~m}$ et l'on pourrait creuser entre eux des souilles n'excédant pas $8 \mathrm{~m}$ de profondeur.

Le Laboratoire de Chatou étudia, sur le modèle fluvial, la conception de tels seuils pour qu'ils résistent aux crues et une solution valable sur ce plan fut élaborée.

Sur le plan de l'hydraulique souterraine, il fut alors demandé à Géohydraulique d'examiner ce que deviendrait la surface piézométrique de la nappe (en période d'étiage) en cas de réalisation de cinq souilles de ce type, séparées par des seuils, s'étendant en amont de Lingostrière jusqu'au pont de la Manda.

Comme il était apparu qu'un abaissement moyen de $3 \mathrm{~m}$ du niveau de la nappe dans cette zone n'aurait pas de conséquences graves, les seuils envisagés seraient arasés à la cote $(0)$ ou au plus $(+1 \mathrm{~m})$ au-dessus du fond moyen du lit en place.

Les premières études de relèvement évoquées précédemment ayant montré que les valeurs de hauteurs piézométriques, fournies par les modèles mathématiques, étaient très voisines de celles données par un modèle simple bidimensionnel en «papier conducteur », il fut décidé de recourir à ce dernier type de modèle pour évaluer l'influence des souilles.

Le modèle analogique «papier conducteur» a reproduit toute la zone comprise entre Lingostrière et la Manda, s'étendant de part et d'autre du Var jusqu'aux coteaux, supposés imperméables. Les souilles, de $900 \mathrm{~m}$ de long, étaient séparées par un seuil de $100 \mathrm{~m}$.

Par ailleurs il était admis, qu'en période d'étiage, le fil d'eau actuel du Var se trouvait à la cote $-1,5 \mathrm{~m}$ par rapport au fond moyen du lit pris pour référence dans la cote d'arasement des seuils.

Les prévisions de variation des hauteurs piézométriques dans la nappe alluviale ont été déterminées pour les différents cas suivants :

- variations des hauteurs piézométriques par rapport à l'état existant:

- seuils supposés arrasés à la cote (0) par rapport au niveau moyen du lit,

- seuils supposés arasés à la cote $(+1 \mathrm{~m})$ par rapport au niveau moyen du lit;

- variations des hauteurs piézométriques par rapport à un abaissement uniforme de $3 \mathrm{~m}$ de la nappe alluviale, qui aurait résulté des dispositions premières arrêtées sur cette zone (extraction uniforme sur $3 \mathrm{~m}$ d'épaisseur):

- seuils arasés à $(0)$,

- seuils arasés à $(+1 \mathrm{~m})$.

Les résultats de ces études sont représentés sur la figure 8 . Ils montrent clairement l'intérêt de la technique d'extraction par souilles par rapport à celle d'une extraction uniforme sur $3 \mathrm{~m}$ d'épaisseur.

\section{3 - Schéma d'aménagement retenu et examen sur le terrain de l'influence des seuils réalisés sur le niveau de la nappe alluviale}

\section{1 - SCHÉMA D'AMÉNAGEMENT RETENU}

A partir des années 1968-1969, où un réseau d'observations avait été mis en place tant dans le cours du Var que dans la nappe alluviale, on a observé une baisse sensible du lit du cours d'eau, dans la zone amont de Lingostrière, provoquée par le transport des matériaux lors de crues depuis cette zone vers la zone aval creusée par les extractions. Corrélativement, le niveau de la nappe baissait en amont de Lingostrière alors qu'il s'élevait en aval, du fait de l'interdiction d'extraction dans cette zone et de l'apport de matériaux par les crues.

De ce fait, il a été décidé :

a) De limiter les constructions de seuils dans la partie inférieure du Var à la seule réalisation de l'ouvrage (seuil $\mathrm{n}^{\circ} 1$ ) prévu à l'extrémité aval sous le pont de la route du bord de mer. Cet ouvrage restait indispensable pour deux raisons :

- éviter le creusement naturel du fond du lit à l'aval;

- protéger les ouvrages de captages de la CGE.

b) De procéder à la réalisation de neuf seuils (numérotés de 2 à 10 de l'aval vers l'amont) distants de $1000 \mathrm{~m}$ pour permettre l'extraction des matériaux par souilles en amont 


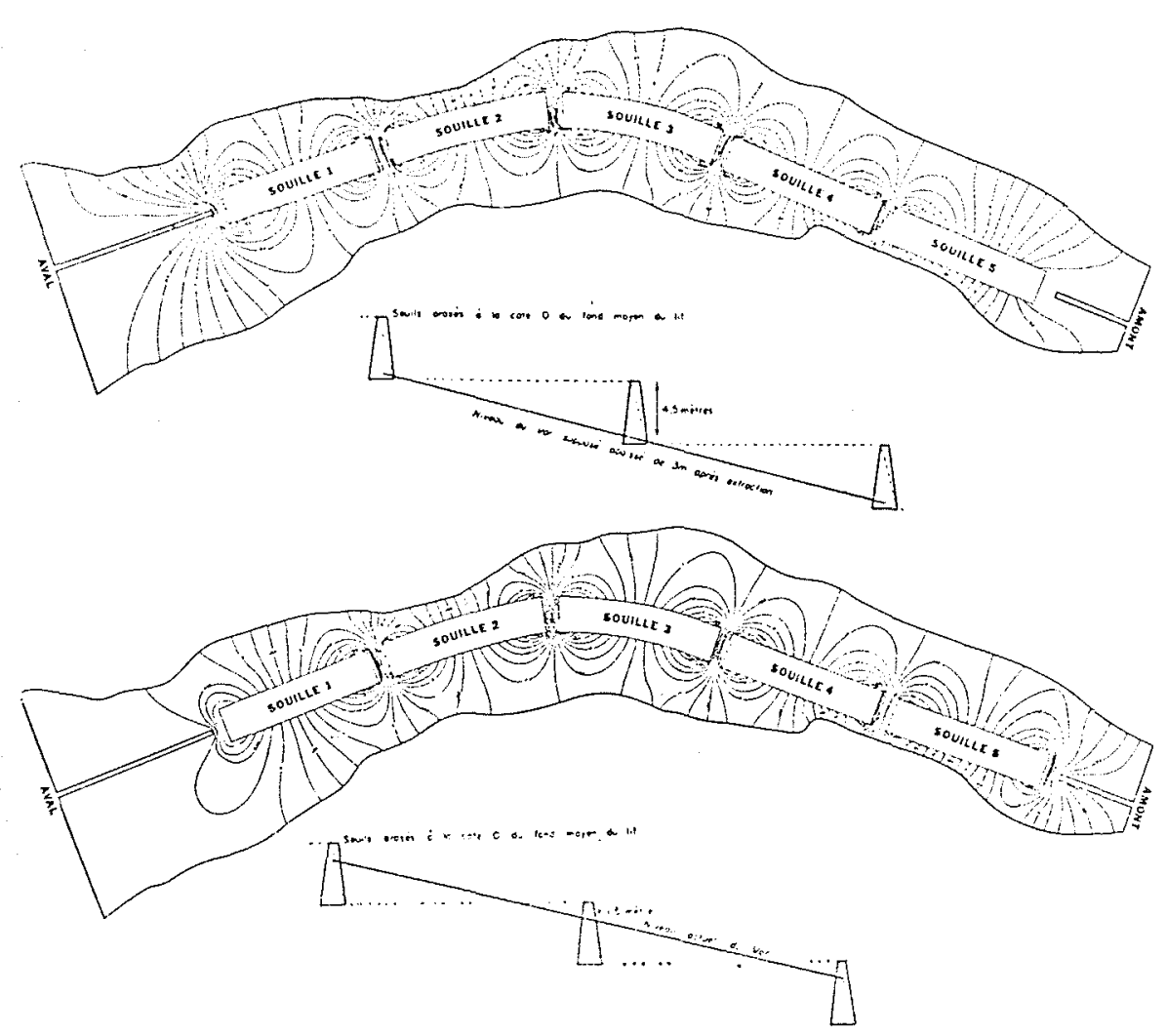

8/ Prévision des effets de l'exploitation contrôlée des graviers par souilles et seuils dans la zone de Lingostrière-La Manda.

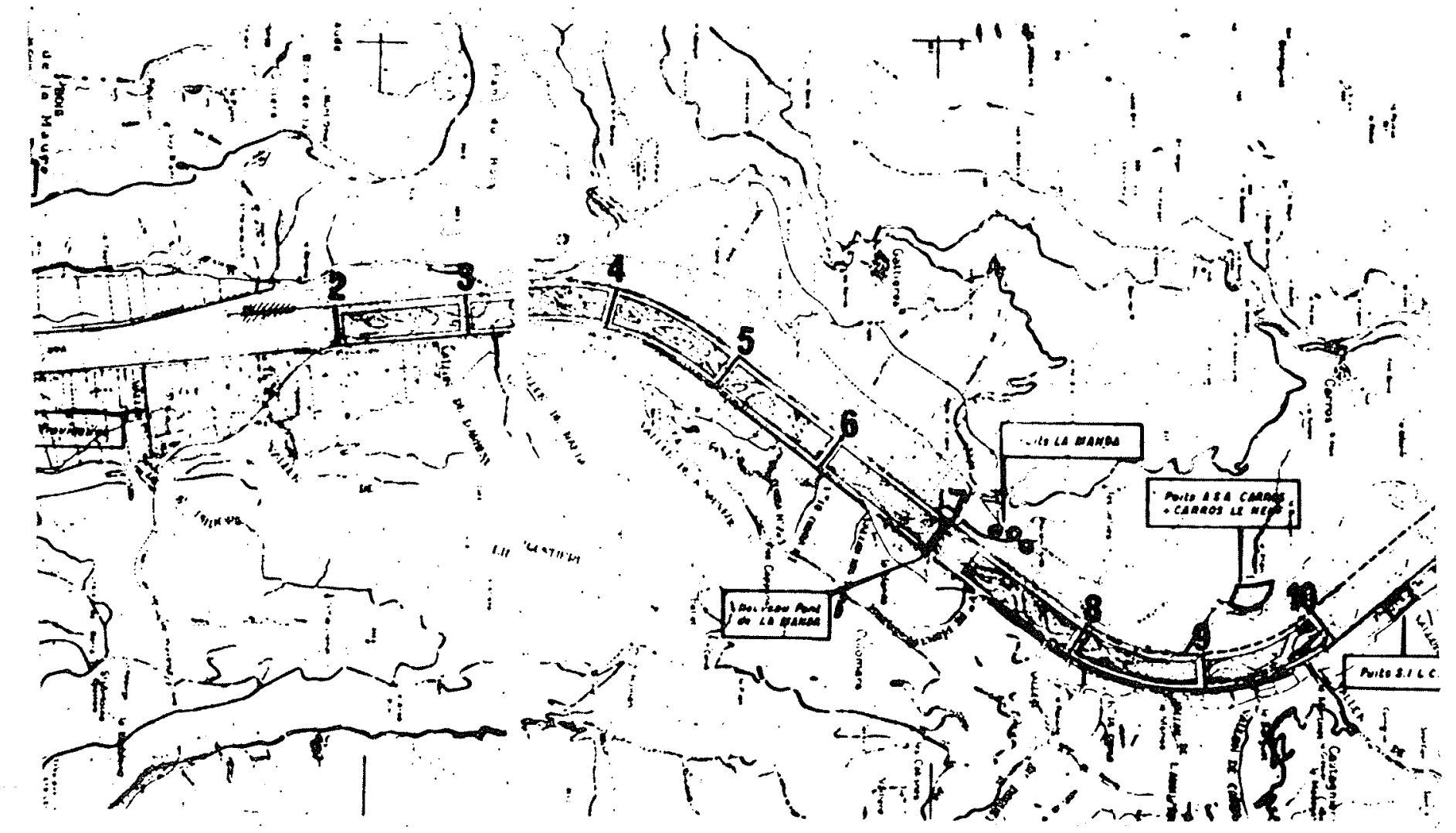

9/ Situation d'ensemble de l'aménagement réalisé. 
de Lingostrière. Ces seuils en enrochements seraient arasés, dans chaque profil, à la cote moyenne du fond du lit relevée en 1968.

A l'heure actuelle, en plus du seuil $\mathrm{n}^{\circ} 1$, cinq seuils sont en service dans la zone d'extraction par souilles.

\section{2 - EXAMEN SUR LE TERRAIN DE L'INFLUENCE DES SEUILS}

Dès 1967, le BRGM était chargé de mettre en place progressivement un réseau d'observations de la nappe et d'assurer la surveillance de la piézométrie. La limitation des crédits n'a pas permis de réaliser immédiatement un réseau très serré d'observations. Toutefois, ces observations apportent des informations précieuses sur l'influence des seuils.

En mai 1970, en particulier, le BRGM a mis en service deux limnigraphes L 16 et L 13 dans les zones des futurs seuils 2 et 3 pour lesquels le démarrage des travaux de construction a eu lieu mi-juin 1970. La position de ces appareils est la suivante:

- par rapport au seuil $n^{\circ} 2$ :

L 16, $90 \mathrm{~m}$ en amont de l'axe du seuil 2, $190 \mathrm{~m}$ de la berge en rive gauche,

L 13, $960 \mathrm{~m}$ en amont de l'axe du seuil 2, $80 \mathrm{~m}$ de la berge en rive gauche;

- par rapport au seuil $n^{\circ} 3$ :

$\mathrm{L} 16,910 \mathrm{~m}$ en aval de l'axe du seuil 3 ,

L 13, $40 \mathrm{~m}$ en aval de l'axe du seuil 3 .

La mise en service du seuil n ${ }^{\circ} 2$ (le plus en aval, situé au niveau de Lingostrière) a été effectuée en février 1971. Elle s'est traduite par les variations piézométriques suivantes:

L 16, relèvement de $1,30 \mathrm{~m}$;

$\mathrm{L} 13$, relèvement de $0,54 \mathrm{~m}$.

La mise en service du seuil $\mathrm{n}^{\circ} 3(1 \mathrm{~km}$ en amont du seuil 2) a été effectuée fin juillet 1971. Elle s'est traduite par les variations piézométriques suivantes :

L 16, relèvement de $0,48 \mathrm{~m}$;

$\mathrm{L} 13$, relèvement de $1,60 \mathrm{~m}$.

Ces relèvements enregistrés témoignent de l'efficacité des seuils et les variations de niveau sont de l'ordre de grandeur de celles qui avaient été prévues par les études théoriques. Il n'est malheureusement pas possible, en l'absence de levés réguliers du fond du lit du Var, de procéder à une comparaison fine des effets observés et des résultats escomptés de ces aménagements : l'étude préalable n'a porté que sur l'état final alors que la construction des seuils s'échelonne sur plusieurs années, l'extraction des graviers se poursuit régulièrement et ses effets sur la nappe se font sentir préalablement à l'aménagement du seuil.

Nous allons, malgré tout, tenter une comparaison globale entre les mesures effectuées et les prévisions de l'étude théorique :

\section{a) Comparaison dans la zone du} limnigraphe L 16 (proximité du seuil no 2) $^{2}$

Les profondeurs du niveau piézométrique moyen de la nappe par rapport au sol, enregistrées au L 16 (cf. rapports BRGM) ont été les suivantes :

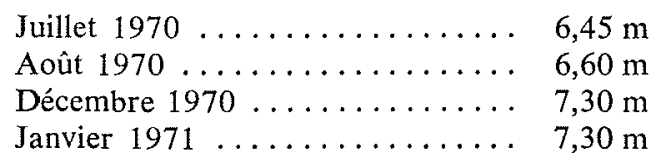

Fermeture seuil 2

Février $1971 \ldots \ldots \ldots \ldots \ldots 6,00 \mathrm{~m}$

Juillet $1971 \ldots \ldots \ldots \ldots \ldots \ldots$ 7,25 m

Fermeture seuil 3

Août $1971 \ldots \ldots \ldots \ldots \ldots \ldots 6,80 \ldots \ldots$

Décembre $1971 \ldots \ldots \ldots \ldots$. $7,50 \mathrm{~m}$

Janvier $1972 \ldots \ldots \ldots \ldots \ldots \ldots$ 7,30 m

On constate qu'entre juillet 1970 et janvier 1971, le niveau piézométrique en $\mathrm{L} 16$ a baissé de l'ordre de $0,8 \mathrm{~m}$. Cet abaissement est certainement dû à l'approfondissement du lit du Var provoqué par les extractions de graviers, largement commencées dans cette zone dès 1970, d'une part, par l'érosion du lit du Var d'autre part.

L'année 1971 a été marquée par l'aménagement des seuils 2 et 3 et par l'exploitation des graviers de toute la souille comprise entre ces deux seuils. Nous pouvons considérer, de ce fait, qu'en fin 1971 la zone du L 16 se trouve dans une situation relativement stable d'aménagement par rapport aux cas théoriques étudiés.

Notons par ailleurs qu'au cours de l'année 1971, le BRGM notait un approfondissement du lit du Var de $2,30 \mathrm{~m}$ au pied du seuil $\mathrm{n}^{\circ} 2$. En ajoutant ces $2,30 \mathrm{~m}$ au $0,8 \mathrm{~m}$ d'abaissement enregistré précédemment, on peut admettre que le fil de l'eau du Var, en aval du seuil $n^{\circ} 2$, s'est affaissé de l'ordre de $3 \mathrm{~m}$ par rapport au niveau existant lors des études: corrélativement, en l'absence de seuils, le niveau piézométrique dans la nappe aurait dû s'abaisser de $3 \mathrm{~m}$. En réalité, entre décembre 1971 et juillet 1970, on enregistre un abaissement limité à $1 \mathrm{~m}$ environ, soit un écart positif de $2 \mathrm{~m}$ dû à la réalisation des seuils. C'est cet écart enregistré de $2 \mathrm{~m}$ qui doit être comparé aux $2,25 \mathrm{~m}$ de relèvement qui résultaient de l'étude sur modèles.

\section{b) Comparaison dans la zone du limnigraphe L 13 (proximité du seuil n" 3 )}

Les profondeurs du niveau piézométrique moyen de la nappe par rapport au sol, enregistrées au L 13 (cf. rapports BRGM) ont été les suivantes :

\begin{tabular}{|c|c|}
\hline Juillet $1970 \ldots \ldots \ldots \ldots \ldots \ldots \ldots$ & $5,4 \mathrm{~m}$ \\
\hline Août $1970 \ldots$ & $5,3 \mathrm{~m}$ \\
\hline Octobre 1970 & $5,7 \mathrm{~m}$ \\
\hline Décembre $1970 \ldots \ldots \ldots \ldots$ & $5,7 \mathrm{~m}$ \\
\hline Fermeture seuil $\mathrm{n}^{\circ} 2$ & \\
\hline Juillet $1971 \ldots \ldots \ldots \ldots \ldots$ & $6,9 \mathrm{~m}$ \\
\hline Fermeture seuil $n^{\circ} 3$ & \\
\hline Août $1971 \ldots \ldots \ldots \ldots \ldots$ & 5,3 \\
\hline Octobre $1971 \ldots \ldots \ldots \ldots \ldots$ & 6,2 \\
\hline Décembre $1971 \ldots \ldots \ldots \ldots$ & 6,4 \\
\hline Janvier $1972 \ldots$ & 6,5 \\
\hline
\end{tabular}

On constate que, dans la zone du L 13, l'abaissement au cours du deuxième semestre 1970 est atténué par rapport à la zone du $\mathrm{L} 16$, les extractions devant être moins importantes dans ce secteur.

Après aménagement des seuils 2 et 3 et pour prendre les mêmes éléments que précédemment, on constate qu'entre 
décembre 1971 et juillet 1970 le niveau piézométrique de la nappe s'est abaissé de $1 \mathrm{~m}$ environ : les études sur modèle avaient prévu un abaissement de $0,80 \mathrm{~m}$ par rapport au niveau d'origine du Var. Nous constatons, là aussi, que les ordres de grandeur sont respectés: le sens dans lequel l'écart est enregistré est, de plus, normal car les niveaux d'eau entre amont et aval du seuil 3 , après aménagement, ont une différence de cote égale à $6,20 \mathrm{~m}$ au lieu des $4,5 \mathrm{~m}$ prévus dans l'étude (de ce fait, le découpage du fil de l'eau par paliers, provoqués par les seuils, est plus écarté du fil de l'eau continu du Var non aménagé).

\section{4 - Observations récentes et conclusions}

La surveillance régulière de la piézométrie de la nappe a permis d'observer une baisse prolongée du niveau de la nappe, même dans la zone comprise entre les seuils 2 et 3 où les extractions sont terminées depuis 1971. Ce phénomène anormal mérite d'être examiné avec une attention particulière, prenant en considération à la fois la pluviométrie enregistrée sur la zone, les pompages effectués dans la nappe et la possibilité d'un éventuel colmatage des souilles après extraction.
Ce dernier aspect, bien qu'il n'altère en rien l'efficacité des seuils quant à leur pouvoir de maintenir le niveau de base de la nappe au-dessus de ce qu'il serait en leur absence, peut en effet influencer le niveau piézométrique de la nappe en réduisant les transferts latéraux naissant dans l'aquifère entre les niveaux amont et aval de part et d'autre des seuils. Par ailleurs, le colmatage affecterait également la réalimentation de la nappe induite à partir du Var lui-même dans les zones où la piézométrie de la nappe s'abaisserait au-dessous du niveau maintenu dans les souilles.

Les services de l'Hydraulique de la Direction Départementale de l'Equipement des Alpes-Maritimes examinent actuellement cette question de très près afin, en cas de colmatage effectif, d'apporter une solution à l'alimentation ainsi perturbée de la nappe. Notamment, les réserves d'eau superficielle occasionnées par l'aménagement des seuils faciliteront la réalisation d'un dispositif de recharge de la nappe alluviale.

En conclusion, nous pensons que cet aménagement du Var, permettant une extraction contrôlée de matériaux nécessaires au développement régional, s'est avéré positif vis-à-vis du maintien de la piézométrie de l'aquifère environnant. Les observations faites à l'issue de la construction des premiers seuils ont montré, par ailleurs, que des outils de prévision valables existaient dans le domaine de l'hydraulique souterraine.

\section{Discussion}

M. le Président remercie M. Prudhomme de sa communication qui montre que l'exploitation des matériaux dans le lit des rivières pose des problèmes différents, mais aussi difficiles à résoudre, que ceux découlant de l'établissement des gravières dans les plaines alluviales.

Il ouvre ensuite la discussion en demandant qu'en raison de l'heure tardive, les questions posées se rapportent très directement au sujet traité.

Avez-yous pu prouver que, dans le secteur considéré, la rivière alimentait bien la nappe avant l'exploitation des gravières? demande M. MORIN (SOGREAH).

Nous n'avons pu obtenir d'observations piézométriques suffisamment fines sur l'ensemble de la nappe, répond $M$. Prudhomme, pour nous rendre exactement compte du sens des échanges. Mais pour ce genre de nappe alluviale, le niveau de base est celui de la rivière. Nous avons cherché à déterminer l'influence du relèvement du niveau de celle-ci sur l'ensemble de la nappe alluviale sans nous attacher à un examen quantitatif des apports.

M. Lefoulon (Inspecteur Général Honoraire EDF) intervient en ces termes:
Il m’apparaît intéressant de rappeler que l'aménagement du Rhin, entre Bâle et Strasbourg, projeté en 1925 , comportait la construction d'un canal latéral au Rhin - dit Grand Canal d'Alsace - implanté sur la rive française et partagé en huit biefs. Le projet a été ainsi exécuté jusqu'à l'application de l'accord franco-allemand de 1956 A partir de Neuf-Brisach il a été prévu à chaque bief :

- le retour au Rhin des eaux turbinées;

- la construction d'un barrage de dérivation;

et, après ce barrage sur la partie asséchée du Rhin (pour maintenir sur la rive allemande la nappe phréatique à la hauteur moyenne d'avant la dérivation):

- la construction de deux ou trois barrages de faible hauteur maintenant le niveau de l'eau dans le lit du Rhin à la cote existant pour le débit moyen du fleuve $\left(1060 \mathrm{~m}^{3} / \mathrm{s}\right)$ avant la réalisation de la dérivation. Par ailleurs, un débit de $15 \mathrm{~m}^{\mathrm{g}} / \mathrm{s}$ a été maintenu dans le lit du fleuve. Ces petits barrages comportent des échelles à poissons et des rampes pour les canoës.

M. le Président remercie M. Prudhomme, M. Morin et M. LefouLON et donne la parole à M. Migniot pour l'exposé de sa communication. 